\title{
Causal relationships between genetically determined metabolites and human intelligence: a Mendelian randomization study
}

\author{
Jian Yang ${ }^{1 \dagger} \mathbb{D}$, Binbin Zhao ${ }^{2 \dagger}$, Li Qian², Fengjie Gao², Yanjuan Fan², Xiaoyan He², Qingyan Ma², Lihong Yang ${ }^{1}$,
} Bin Yan', Wei Wang ${ }^{2}$ and Xiancang $\mathrm{Ma}^{2,3^{*}}$

\begin{abstract}
Intelligence predicts important life and health outcomes, but the biological mechanisms underlying differences in intelligence are not yet understood. The use of genetically determined metabotypes (GDMs) to understand the role of genetic and environmental factors, and their interactions, in human complex traits has been recently proposed. However, this strategy has not been applied to human intelligence. Here we implemented a two-sample Mendelian randomization (MR) analysis using GDMs to assess the causal relationships between genetically determined metabolites and human intelligence. The standard inverse-variance weighted (IVW) method was used for the primary MR analysis and three additional MR methods (MR-Egger, weighted median, and MR-PRESSO) were used for sensitivity analyses. Using 25 genetic variants as instrumental variables (IVs), our study found that 5-oxoproline was associated with better performance in human intelligence tests $\left(P_{I V W}=9.25 \times 10^{-5}\right)$. The causal relationship was robust when sensitivity analyses were applied $\left(P_{\text {MR-Egger }}=0.0001, P_{\text {Weighted median }}=6.29 \times 10^{-6}, P_{\text {MR-PRESSO }}=0.0007\right)$, and repeated analysis yielded consistent result $\left(\mathrm{P}_{\mathrm{IVW}}=0.0087\right)$. Similarly, also dihomo-linoleate $(20: 2 \mathrm{n6})$ and $\mathrm{p}$-acetamidophenylglucuronide showed robust association with intelligence. Our study provides novel insight by integrating genomics and metabolomics to estimate causal effects of genetically determined metabolites on human intelligence, which help to understanding of the biological mechanisms related to human intelligence.
\end{abstract}

Keywords: Genetically determined metabolite, Human intelligence, Mendelian randomization, Metabolic pathway, 5-Oxoproline

\section{Introduction}

Intelligence affects all aspects of human life [1]. During the school years, some individuals show higher intelligence, attain better marks in exams, and have better prospects for further education $[2,3]$. In the workplace, intelligence influences performance, efficiency, the ability to cope with difficulties, and career achievements [4]. Intelligence is also a predictor of higher quality of life

\footnotetext{
*Correspondence: maxiancang@163.com

†Jian Yang and Binbin Zhao have contributed equally to this work

${ }^{2}$ Department of Psychiatry, the First Affiliated Hospital of Xi'an Jiaotong University, No. 277 Yanta West Road, Xi'an 700061, China

Full list of author information is available at the end of the article
}

and better health outcomes [5, 6]. Revealing the biological bases of individual differences in human intelligence has become a central and enduring aim of psychological and brain sciences. During the past decade, advances in genetic research have greatly promoted our understanding of intelligence [7-10]. However, further insight on its biological basis is needed.

Understanding the role of genetic characteristics and their interaction with environmental factors is the key to reveal the biological mechanisms underlying differences in human intelligence [11]. Currently, omics technologies (such as genomics, metabolomics, etc.) are widely used to provide a comprehensive characterization at the 
molecular level of the human body as a biological system. These approaches have successfully identified a number of informative biomarkers and greatly advanced our knowledge of the molecular mechanisms responsible for many traits. However, most omics studies focus only on a single layer, and therefore fail to capture information across multiple omics assays [12]. Recently, researchers have linked metabolomics traits to genomic information through genome-wide association studies (GWAS) on non-targeted metabolic profiling [13-15]. A large database of genetically determined metabotypes (GDMs) has been thus established to provide comprehensive insights of how genetic variation influences metabolism [16]. The established GDMs provide important intermediates to reveal the role of the interactions between genetics and metabolic traits in determining differences in human intelligence.

Mendelian randomization (MR) is a novel genetic epidemiology study design using genetic variants as instrumental variables (IVs) to investigate whether a modifiable exposure is causally related to a medically relevant disease risk [17]. The fundamental assumption utilized in the MR framework is that if genetic variants essentially affect the biological effects of a modifiable exposure, they should be also related to the exposure-related disease risk. Exploiting the fact that inherent genetic variants are not generally susceptible to environmental variables, the MR design can avoid the potential confounding factors that are common in conventional observational studies [18]. In recent years, the explosion in the number of published GWAS summary data has increased the popularity of MR approaches (and in particular of two-sample MR analysis) as tools to infer the causality of risk factors on complex health outcomes [19-21]. In this study, using GDMs and the results of GWAS on intelligence, we implement two-sample MR analysis to: (1) assess the causal effects of genetically determined metabolites on human intelligence; (2) investigate the genetic basis that may play a central role in determining the variation of the related metabolites and the differences in human intelligence; (3) identify potential metabolic pathways involved in the biological processes related to intelligence.

\section{Methods}

\section{GWAS scans with metabolomics traits}

Shin et al. reported the most comprehensive exploration of genetic influences on human metabolism so far, by performing a GWAS of non-targeted metabolomics on 7824 healthy adults. [16]. Metabolic profiling was carried out on fasting serum using high-performance liquid chromatography and gas chromatography separation coupled with tandem mass spectrometry. After quality control, 486 metabolites were retained for genetic analysis, among which 309 were chemically identified and could be further assigned to 8 metabolic groups (amino acids, carbohydrates, cofactors and vitamins, energy, lipids, nucleotides, peptides, and xenobiotics), while the other 177 were classified as 'unknown. The final genomewide association analyses were carried out on approximately 2.1 million single nucleotide polymorphisms (SNPs). Full summary statistics for the 486 metabolites can be found at the Metabolomics GWAS Server (http:// metabolomics.helmholtz-muenchen.de/gwas/).

\section{IVs for the $\mathbf{4 8 6}$ metabolites}

The foundational principle of MR relies on the existence of valid IVs. A genetic variant is a valid IV if it is (i) significantly associated with the exposure, (ii) independent of confounders, and (iii) associated with the outcome only through the exposure [22]. To identify valid IVs, we first selected the SNPs with significance $P<1 \times 10^{-5}$, so as to account for a proportion as large as possible of the variance explained for the corresponding metabolite. We next performed a clumping procedure (linkage disequilibrium threshold of $\mathrm{r}^{2}<0.1$ within a $500-\mathrm{kb}$ window) to select the independent SNPs using the PLINK software (v1.9). To avoid the negative impact of weak IVs, we further used the proportion of variation explained by each IV $\left(R^{2}\right)$ and the $F$ statistics to select SNPs strong enough to be valid IVs. Typically, an $F$ statistic $>10$ is considered sufficient for MR analysis [23].

\section{GWAS summary data on intelligence}

GWAS summary statistics for intelligence were obtained from the study by Savage et al. [10]. Briefly, these authors performed a large GWAS meta-analysis of 269,867 individuals from 14 cohorts of European ancestry. Intelligence was assessed using different neurocognitive tests and the general factor of intelligence (Spearman's g). Although differences in assessment methods might reduce the power to detect associations in meta-analyses, this approach can at the same time reduce type I errors by removing measurement errors, and therefore identify SNPs with robust associations to the common latent factor underlying intelligence across different methods. Stringent quality control procedures were applied to the summary statistics for each cohort. Association analysis was conducted controlling for covariates of age, sex, genotyping array, socioeconomic status for specific cohort, and twenty European-based ancestry principal components. Finally, a total of 9,295,118 SNPs were included in the meta-analysis.

\section{Statistical analysis}

Primary two-sample MR analyses were performed using the standard inverse-variance weighted (IVW) 
method. The IVW method provides a consistent estimate of causal effects by combining the ratio estimates of each variant in a fixed-effect meta-analysis model [23]. The P-value was calculated with a standard normal cumulative distribution function on the ratio of the combined causal effect and its standard error. The significance threshold to declare a causal relationship for the IVW-based MR estimate was set, using Bonferroni correction, at $\mathrm{P}<1.03 \times 10^{-4}(=0.05 / 486)$. Associations with $\mathrm{P}<0.05$, but not reaching the Bonferroni-corrected threshold, were reported as suggestive of association.

The IVW method provides an unbiased estimate under the assumption that all genetic variants are valid IVs. However, this assumption is easily violated, leading to inaccurate estimates, when horizontal pleiotropy occurs (some variants act on the outcome via a different intermediary) [24]. To avoid the effects of widespread horizontal pleiotropy in MR, we further performed sensitivity analyses using three additional MR methods: the MR-Egger method, which provides a consistent causal effect estimate, even when all genetic variants violate the assumptions defining valid IVs, under a weaker assumption (known as the InSIDE [instrument strength independent of direct effect] assumption) [24]; the weighted median method, which introduces a weighted median estimator and provides a more precise estimate than MR-Egger regression without the InSIDE assumption [25]; and the MR-PRESSO method, a newly developed approach which can identify and correct for horizontal pleiotropic outliers in MR [26]. We further used the MR-PRESSO global test as well as the intercept of the MR-Egger regression to test for pleiotropy, and we also evaluated heterogeneity with the $I^{2}$ and the Cochran Q test. Typically, $I^{2}>25 \%$ or Cochran Q-derived $P<0.05$ were used as indicators of possible horizontal pleiotropy. Analyses were carried out using the packages MendelianRandomization and MR-PRESSO in R (version 3.6.1).

\section{Replication}

We next used GWAS datasets of four other related outcomes to replicate the findings of our MR estimates. The first dataset was obtained from another GWAS of intelligence with 248,482 samples from the UK Biobank [27]. Summary statistics of cognitive performance $(\mathrm{n}=257,828)$ and educational attainment $(\mathrm{n}=766,345)$ were obtained from the study of Lee et al. [28]. Genetic associations with income $(n=286,301)$ were extracted from the large publicly available Lothian Birth Cohorts of 1921 and 1936 data-sharing resource [29]. Notably, the Davies et al. reported another GWAS for intelligence with a larger sample size, but the summary data for full dataset is not available due to data permissions [30].

\section{Metabolic pathway analysis}

Metabolic pathway analysis was carried out using the web-based tool suite MetaboAnalyst 4.0 (https://www. metaboanalyst.ca/) [31]. For this analysis, we extracted all metabolites showing suggestive associations in the IVW estimates $\left(\mathrm{P}_{\mathrm{IVW}}<0.05\right)$. Two libraries of metabolic pathways or metabolite sets were selected for enrichment analysis, namely the Small Molecule Pathway Database (SMPDB, http://www.smpdb.ca) [32] and the Kyoto Encyclopedia of Genes and Genomes (KEGG, https:// www.kegg.jp/) database [33]. P-values $<0.05$ were considered statistically significant.

\section{Results \\ Causal effects of the metabolites on intelligence}

We selected 3-675 independent genetic variants as IVs for each of the 486 metabolites (Additional file 4: Table S1). On average, the IVs explained $4.7 \%$ (range $0.8-83.5 \%)$ of the variance of their respective metabolic traits. The minimum $\mathrm{F}$ statistic used to evaluate the strength of these IVs was 20.33. Using these IVs, IVW identified 16 known metabolites and 16 unknown metabolites that might have causal effects on human intelligence (Fig. 1, Additional file 4: Table S2). Among the 16 known metabolic traits, 5-oxoproline was significantly associated with intelligence after Bonferroni correction $\left(\mathrm{P}_{\mathrm{IVW}}=9.25 \times 10^{-5}\right)$. Using $25 \mathrm{SNPs}$ as proxy, we observed a 0.24 increase in the score of the Spearman's $g$ test for an increase of one standard deviation (SD) in the level of 5-oxoproline ( $\beta=2.10 ; 95 \%$ Confidence interval $[\mathrm{CI}] 0.12$ to 0.35 ). We also found 15 other metabolites to be suggestive for association, including indolelactate $\left(\beta=-0.09 ; 95 \% \mathrm{CI}-0.81\right.$ to $\left.-0.01, \mathrm{P}_{\mathrm{IVW}}=0.0313\right)$, mannitol $\left(\beta=-0.03 ; 95 \% \mathrm{CI}-0.06\right.$ to $\left.-0.01, \mathrm{P}_{\mathrm{IVW}}=0.0223\right)$, and 2-oleoylglycerophosphocholine $(\beta=0.18 ; 95 \% \mathrm{CI}$ 0.05 to $0.30, \mathrm{P}_{\mathrm{IVW}}=0.0055$ ).

\section{Sensitivity analysis}

Table 1 shows the results of the sensitivity analyses for the 16 IVW-identified known metabolites. The causal relationship between 5-oxoproline and intelligence was robust when additional MR methods were applied $\left(\mathrm{P}_{\mathrm{MR} \text {-Egger }}=0.0001, \mathrm{P}_{\text {Weighted median }}=6.29 \times 10^{-6}\right.$, $\left.\mathrm{P}_{\text {MR-PRESSO }}=0.0007\right)$, and no horizontal pleiotropy was observed $\left(\mathrm{P}_{\text {Intercept }}=0.09, \mathrm{P}_{\text {Global test }}=0.06, \quad I^{2}=25 \%\right.$, $\left.\mathrm{P}_{\text {Heterogeneity }}=0.13\right)$. Two other metabolites also showed robust associations with intelligence, namely dihomo-linoleate $(20: 2 \mathrm{n} 6) \quad\left(\mathrm{P}_{\text {MR-Egger }}=0.0494, \mathrm{P}_{\text {Weighted }}\right.$ median $=0.0236, \quad P_{\text {MR-PRESSO }}=0.0293, \quad P_{\text {Global test }}=0.16$ ) 


\begin{tabular}{|c|c|c|c|c|c|}
\hline Metabolites & SNPs & $\beta(95 \% \mathrm{Cl})$ & Lower Risk & Higher Risk & $P$ Value \\
\hline \multicolumn{6}{|l|}{ Amino acid } \\
\hline 5-oxoproline & 25 & $0.24(0.12$ to 0.35$)$ & & $\longrightarrow$ & $9.25 \mathrm{E}-05$ \\
\hline indolelactate & 17 & $-0.09(-0.18$ to -0.01$)$ & & & 0.0313 \\
\hline \multicolumn{6}{|l|}{ Carbohydrate } \\
\hline mannitol & 13 & $-0.03(-0.06$ to -0.01$)$ & & & 0.0223 \\
\hline \multicolumn{6}{|l|}{ Lipid } \\
\hline 2-oleoylglycerophosphocholine & 16 & $0.18(0.05$ to 0.30$)$ & & $\longrightarrow$ & 0.0055 \\
\hline 2-palmitoylglycerophosphocholine & 24 & $0.14(0.04$ to 0.25$)$ & & $\longrightarrow$ & 0.0062 \\
\hline 2-stearoylglycerophosphocholine & 13 & $0.11(0.03$ to 0.20$)$ & & $\rightarrow-$ & 0.0114 \\
\hline 1-oleoylglycerol (1-monoolein) & 16 & $0.09(0.01$ to 0.17$)$ & & - & 0.0359 \\
\hline dihomo-linoleate $(20: 2 n 6)$ & 9 & $-0.14(-0.25$ to -0.04$)$ & $\longrightarrow$ & & 0.0081 \\
\hline docosapentaenoate (n3 DPA; 22:5n3) & 11 & $-0.16(-0.29$ to -0.03$)$ & $\longrightarrow$ & & 0.0194 \\
\hline linolenate $(18: 3 n 3$ or 6$)$ & 5 & $-0.20(-0.38$ to -0.03$)$ & $\rightarrow-$ & & 0.0205 \\
\hline acetylcarnitine & 19 & $-0.25(-0.40$ to -0.10$)$ & $\longrightarrow$ & & 0.0011 \\
\hline \multicolumn{6}{|l|}{ Peptide } \\
\hline cyclo(leu-pro) & 15 & $-0.06(-0.12$ to -0.01$)$ & & & 0.0267 \\
\hline \multicolumn{6}{|l|}{ Xenobiotics } \\
\hline stachydrine & 7 & $0.06(0.02$ to 0.11$)$ & & 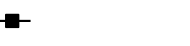 & 0.0050 \\
\hline p-acetamidophenylglucuronide & 63 & $0.01(0.00$ to 0.01$)$ & & & 0.0411 \\
\hline salicyluric glucuronide & 13 & $-0.01(-0.02$ to -0.01$)$ & & & 0.0192 \\
\hline \multirow[t]{3}{*}{ hydroquinone sulfate } & 17 & $0.98(0.95,0.99)$ & \multicolumn{2}{|c|}{ 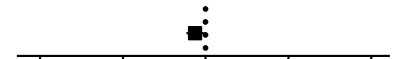 } & 0.0208 \\
\hline & & & $\begin{array}{ll}-0.4 & -0.2\end{array}$ & $0.2 \quad 0.4$ & \\
\hline & & \multicolumn{4}{|c|}{$\begin{array}{l}\text { Effects of Genetically Determined } \\
\text { Metabolites on Intelligence }\end{array}$} \\
\hline
\end{tabular}

and p-acetamidophenylglucuronide $\left(\mathrm{P}_{\mathrm{MR} \text {-Egger }}=0.0075\right.$, $\mathrm{P}_{\text {Weighted median }}=0.0060, \quad \mathrm{P}_{\text {MR-PRESSO }}=0.0454, \quad \mathrm{P}_{\text {Global }}$ test $=0.0611$, and there were no evidence of horizontal pleiotropy $\left(\mathrm{P}_{\text {Intercept }}=0.24, \mathrm{P}_{\text {Global test }}=0.17\right.$, $I^{2}=0 \%, \quad \mathrm{P}_{\text {Heterogeneity }}=0.96 \quad$ for dihomo-linoleate $(20: 2 \mathrm{n} 6)$ and $\mathrm{P}_{\text {Intercept }}=0.06, \mathrm{P}_{\text {Global test }}=0.06, I^{2}=17 \%$, $\mathrm{P}_{\text {Heterogeneity }}=0.13$ for $\mathrm{p}$-acetamidophenylglucuronide; Table 1). Funnel plots appeared generally symmetrical for all the three metabolites, also suggesting no evidence for horizontal pleiotropy (Additional file 1: Fig. S1). Dihomolinoleate $(20: 2 \mathrm{n} 6)$ showed a negative association with intelligence $\left(\beta_{\mathrm{IVW}}=-0.14 ; 95 \% \mathrm{CI}-0.25\right.$ to -0.04$)$, while the association between p-acetamidophenylglucuronide and intelligence was positive $\left(\beta_{\mathrm{IVW}}=0.01 ; 95 \% \mathrm{CI}\right.$ 0.00 to 0.01 ). The causal association between 5 -oxoproline and human intelligence is shown on Fig. 2, while the associations for dihomo-linoleate $(20: 2 \mathrm{n} 6)$ and p-acetamidophenylglucuronide with intelligence are represented on Fig. 3. Notably, the very small effect size for p-acetamidophenylglucuronide on intelligence might limit its potential utility as a biomarker.

\section{Associations with other relevant outcomes}

We next repeated the main findings using summary statistics from other data sources. Figure 4 showed the results of causal effects of 5-oxoproline on human intelligence from another data source, cognitive performance, educational attainment, and income. The effect of genetically determined 5-oxoproline on intelligence (Replication) was similar $(\beta=0.17 ; 95 \%$ CI 0.04 to 0.30 , $\left.\mathrm{P}_{\mathrm{IVW}}=0.0087\right)$ to the result of initial MR estimates, and the causal associations were robust when different methods were performed $\left(P_{\text {Weighted median }}=0.0003\right.$, $\mathrm{P}_{\text {MR-Egger }}=0.0035$ ). The results also showed that 5-oxoproline was significantly associated with cognitive performance $\left(\mathrm{P}_{\mathrm{IVW}}=0.0001, \mathrm{P}_{\text {Weighted median }}=1.44 \times 10^{-6}\right.$, $\left.\mathrm{P}_{\text {MR-Egger }}=0.0009\right)$. However, no evidences for association were found between 5-oxoproline and educational attainment $\quad\left(\mathrm{P}_{\mathrm{IVW}}=0.5595, \mathrm{P}_{\text {Weighted median }}=0.3417\right.$, $\left.\mathrm{P}_{\text {MR-Egger }}=0.4611\right)$, as well as income $\left(\mathrm{P}_{\mathrm{IVW}}=0.7854\right.$, $\left.\mathrm{P}_{\text {Weighted median }}=0.4287, \mathrm{P}_{\text {MR-Egger }}=0.6178\right)$. Besides, the effects of dihomo-linoleate $(20: 2 \mathrm{n} 6)$ and p-acetamidophenylglucuronide on intelligence were also significant 


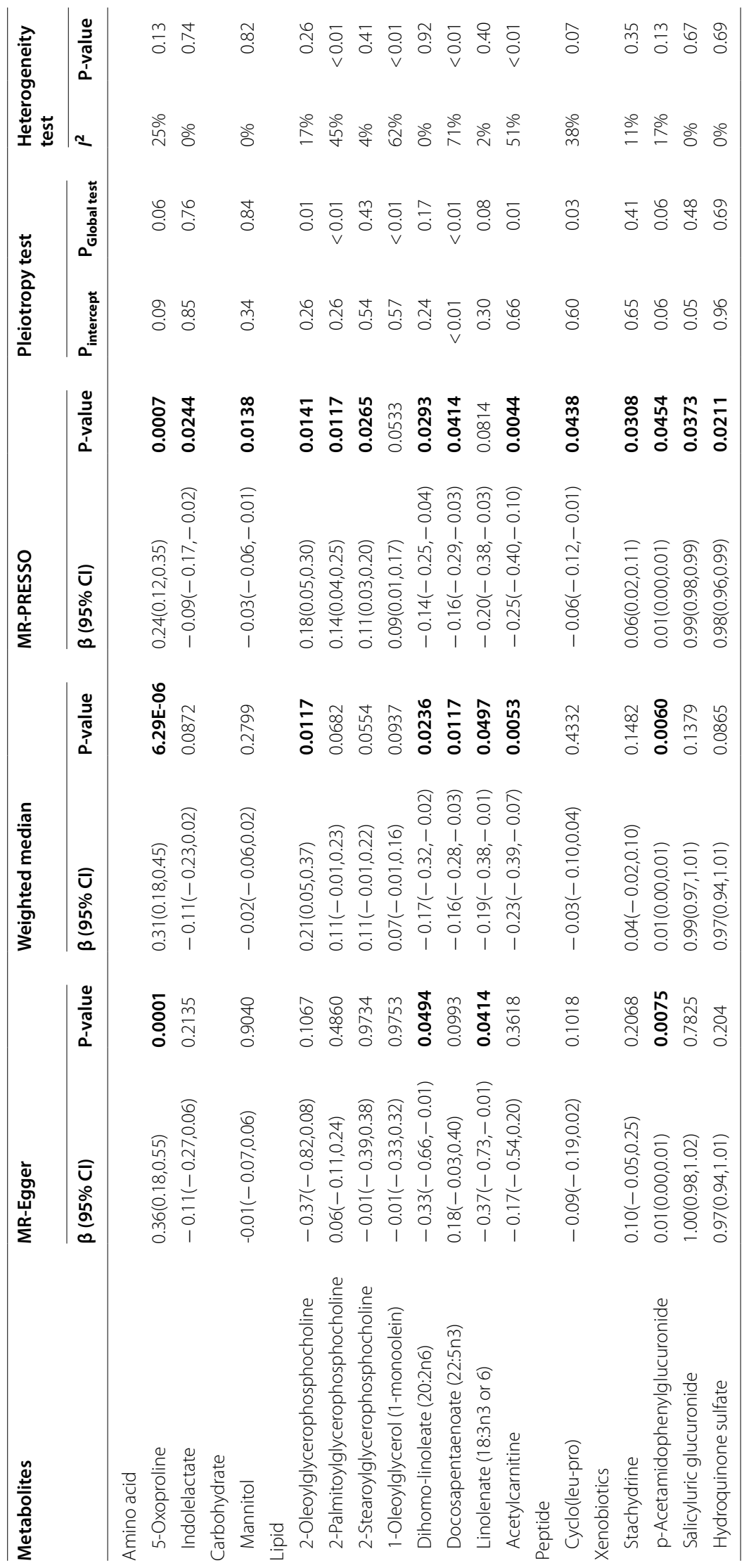




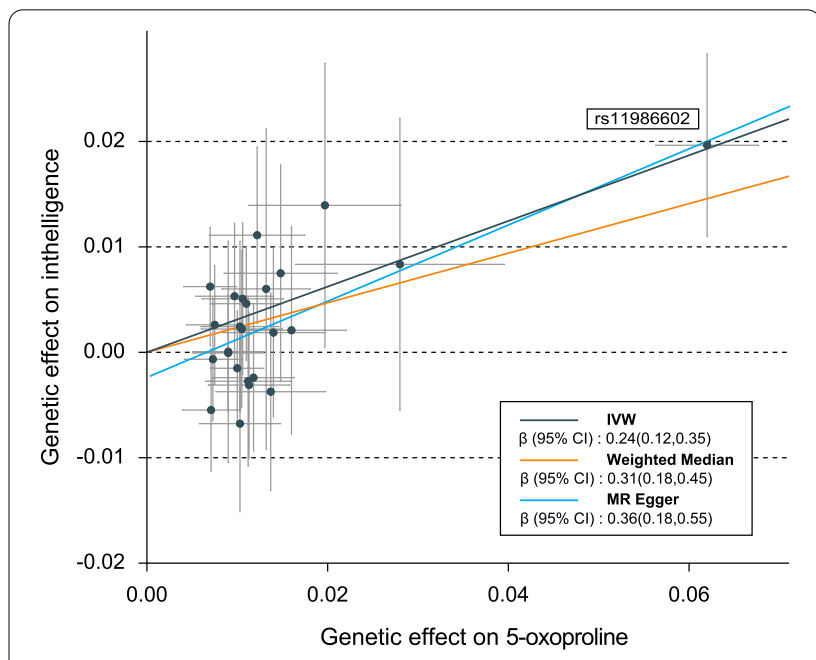

Fig. 2 Genetic associations between 5-oxoproline and intelligence

in the replication stage (Additional file 2: Fig. S2; Additional file 3: Fig. S3).

\section{Genetic basis for the causal associations}

We further investigated the genetic variants that affected both metabolite levels and intelligence. Table 2 shows the 25 SNPs used as IV of 5-oxoproline. Among them, rs11986602 showed the most significant association with 5-oxoproline $(\beta=-0.0620 ; \mathrm{SE}=0.0029$, $\left.\mathrm{P}=6.29 \times 10^{-104}\right)$. Notably, it also showed a strong association signal with intelligence $(\beta=-0.0196 ; \mathrm{SE}=0.0044$, $\left.\mathrm{P}=9.53 \times 10^{-6}\right)$. Moreover, this SNP had the largest effect sizes on both 5-oxoproline and intelligence, suggesting that the related genetic locus might provide valuable information on the biological mechanisms of intelligence, and that 5-oxoproline might be an important functional intermediate to understand the biological process through which genetics affects intelligence. The IVs for dihomo-linoleate (20:2n6) and p-acetamidophenylglucuronide are shown in Additional file 4: Tables S3 and S4.

\section{Metabolic pathway analysis}

Table 3 shows the results of the metabolic pathway analysis. Based on the 16 known metabolites identified by the IVW method, we detected only one significant metabolic pathway associated with intelligence, namely Alpha linolenic acid and linoleic acid metabolism $(\mathrm{P}=0.0062)$. Two metabolites identified by IVW, docosapentaenoate (n3 DPA; 22:5n3) and linolenate (18:3n3 or 6), are involved in Alpha linolenic acid and linoleic acid metabolism according to the SMPDB database. Importantly, many of the metabolites found by our analysis have not been assigned to any metabolic pathway currently recorded in the SMPDB or KEGG databases. Extensive further research will be needed to explore whether these metabolites are involved in biological processes relevant to differences in human intelligence.

\section{Discussion}

We implemented a two-sample MR analysis to assess the causal relationships between genetically determined metabolites and human intelligence. Using genetic variants as IVs, we found that the genetically determined levels of 5-oxoproline were associated with better performance in human intelligence tests. This causal
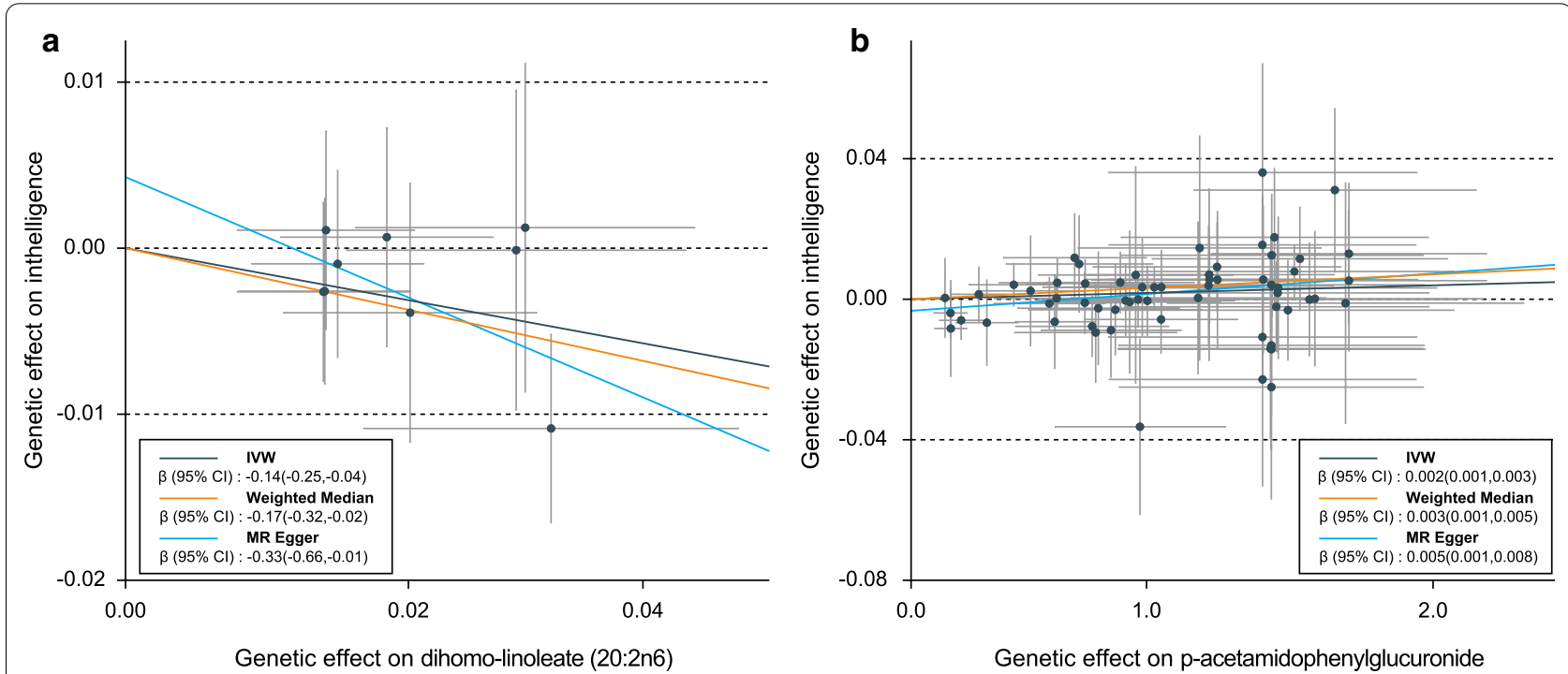

Fig. 3 Genetic associations of two suggestive metabolites with intelligence. a Dihomo-linoleate (20:2n6); b p-acetamidophenylglucuronide 


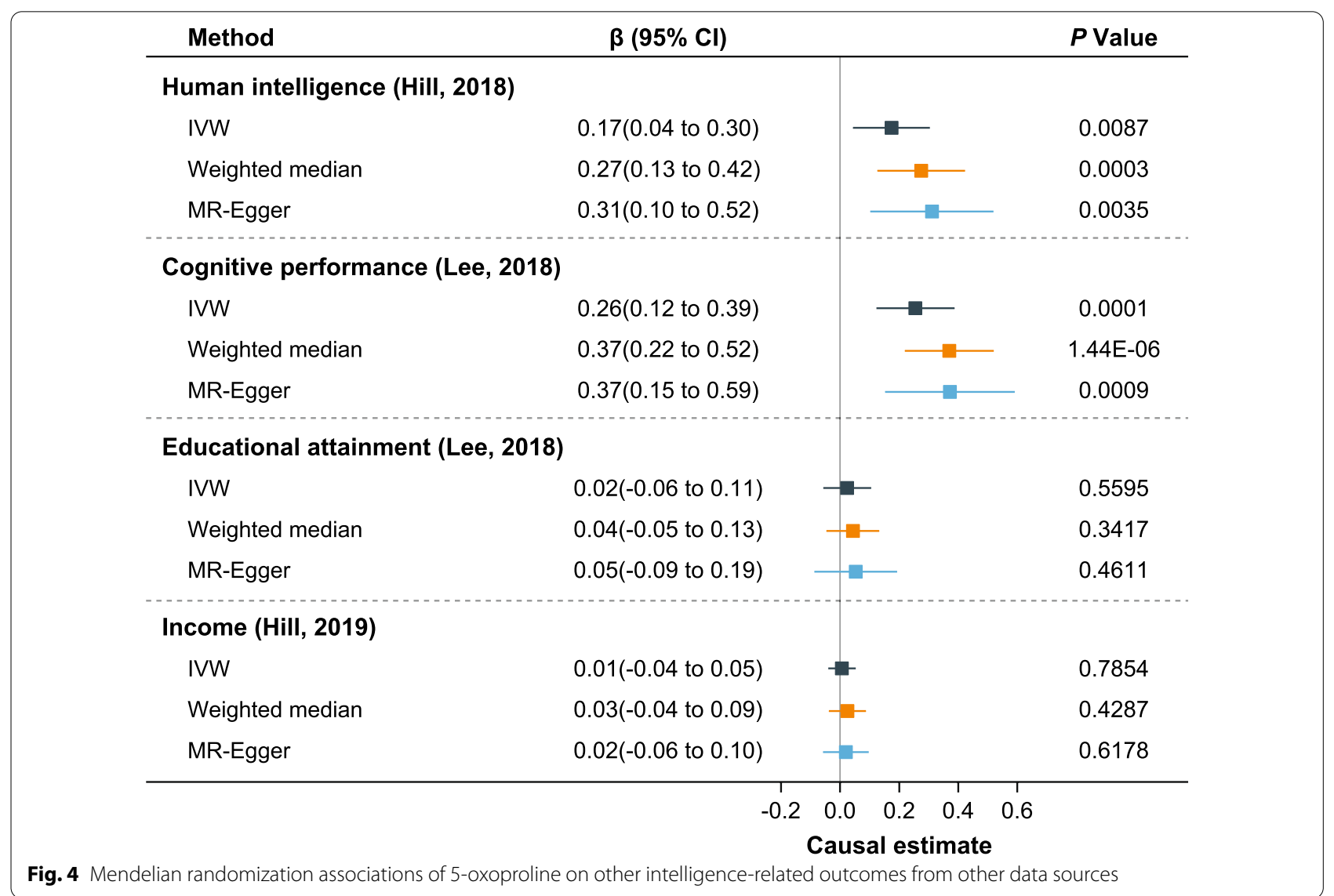

association was not affected by confounders such as educational attainment and household income, and was well replicated using samples from other data source. Our study also identified other metabolites and metabolic pathways involved in biological processes related to human intelligence, such as dihomo-linoleate $(20: 2 n 6)$ and p-acetamidophenylglucuronide. To the best of our knowledge, this is the first study combining information from genomics and metabolomics to assess the causal effects of metabolome traits on human intelligence.

5-Oxoproline, also known as pyroglutamic acid, is a cyclized derivative of L-glutamic acid that participates substantially in the glutamate and glutathione metabolism [34]. Disturbances in glutamate and glutathione metabolism can lead to a series of neurologic phenotypes, including developmental delay, ataxia, seizures, and intellectual disability [35]. Moreover, 5-oxoproline was also developed and sold as an over-the-counter "smart drug" for cognitive and memory improvement [36, 37]. However, it was also demonstrated that metabolic acidosis could be caused by excessive 5-oxoproline generation, with multiple adverse effects on many organ systems [38]. Our study found that elevated levels of 5-oxoproline were associated with a higher score in intelligence tests, supporting the potential usefulness of 5-oxoproline in improving intelligence-related performance. However, more work aimed at understanding the molecular mechanisms involved is needed to further clarify the role of this compound in human intelligence.

Genetic factors played a central role in our study of the causal relationship between metabolic traits and intelligence. The SNP rs11986602 (corresponding to the EXOSC4 gene) was the most significantly associated to both 5-oxoproline levels and human intelligence. Although rarely discussed in the past literature, EXOSC4 is known to be related to the protein kinase $\mathrm{R}$ (PKR)-like endoplasmic reticulum kinase (PERK, encoded by the EIF2AK3 gene), which regulates gene expression [39]. A recent study reported that locally reduced PERK expression or activity could enhance neuronal excitability and improve memory and cognitive function in young mice [40]. Another study provided evidence that PERK is a key regulator of memory impairments and neurodegeneration in Alzheimer's disease [41]. Thus, EXOSC4 might be a causal risk gene participating in physiological processes important for human intelligence.

We further focused on the metabolic pathways that might be involved in the biological processes associated 
Table 2 Genetic predictors of 5-oxoproline and their association with Intelligence

\begin{tabular}{|c|c|c|c|c|c|c|c|c|c|c|}
\hline \multirow[t]{2}{*}{ SNP } & \multirow[t]{2}{*}{ Gene } & \multirow[t]{2}{*}{$\mathrm{CHR}$} & \multirow[t]{2}{*}{$\mathrm{A} 1$} & \multirow[t]{2}{*}{$\mathrm{A} 2$} & \multicolumn{3}{|c|}{ 5-oxoproline } & \multicolumn{3}{|c|}{ Intelligence } \\
\hline & & & & & Beta & SE & $P$ value & Beta & SE & $P$ value \\
\hline rs11986602 & EXOSC4 & 8 & A & $\mathrm{T}$ & -0.0620 & 0.0029 & 1.07E-104 & -0.0196 & 0.0044 & $9.53 \mathrm{E}-06$ \\
\hline rs9987070 & - & 7 & C & G & -0.0280 & 0.0059 & $2.43 \mathrm{E}-06$ & -0.0083 & 0.0071 & 0.2381 \\
\hline rs10890517 & - & 2 & $\mathrm{~T}$ & $C$ & -0.0197 & 0.0043 & $3.45 \mathrm{E}-06$ & -0.0139 & 0.0069 & 0.0427 \\
\hline rs5764925 & - & 22 & A & G & -0.0160 & 0.0031 & $1.91 \mathrm{E}-07$ & -0.0021 & 0.0050 & 0.6782 \\
\hline rs13159409 & - & 5 & $\mathrm{~T}$ & G & -0.0148 & 0.0032 & $2.74 \mathrm{E}-06$ & -0.0075 & 0.0053 & 0.1535 \\
\hline rs12294182 & MICAL2 & 11 & $\mathrm{~T}$ & $C$ & 0.0140 & 0.0029 & $1.07 E-06$ & 0.0019 & 0.0041 & 0.6478 \\
\hline rs2068157 & AACSP1 & 5 & $\mathrm{~T}$ & $C$ & 0.0137 & 0.0031 & $8.99 \mathrm{E}-06$ & -0.0037 & 0.0048 & 0.4362 \\
\hline rs9964014 & DLGAP1 & 18 & $\mathrm{~T}$ & $C$ & -0.0132 & 0.0025 & $1.80 \mathrm{E}-07$ & -0.0060 & 0.0078 & 0.4388 \\
\hline rs11605366 & - & 11 & $\mathrm{~T}$ & $C$ & -0.0122 & 0.0027 & $7.55 \mathrm{E}-06$ & -0.0111 & 0.0043 & 0.0094 \\
\hline rs12143589 & - & 1 & A & G & 0.0118 & 0.0023 & $3.38 \mathrm{E}-07$ & -0.0024 & 0.0036 & 0.5034 \\
\hline rs13013224 & LOC105369165 & 2 & C & G & 0.0113 & 0.0023 & $7.04 \mathrm{E}-07$ & -0.0031 & 0.0035 & 0.3781 \\
\hline rs306676 & - & 13 & A & G & 0.0112 & 0.0024 & $2.48 \mathrm{E}-06$ & -0.0027 & 0.0041 & 0.5047 \\
\hline rs9650466 & $\mathrm{MROH} 1$ & 8 & $\mathrm{~T}$ & $C$ & 0.0110 & 0.0020 & $3.80 E-08$ & 0.0046 & 0.0028 & 0.0938 \\
\hline rs1001210 & ATXN1 & 6 & $\mathrm{~T}$ & $C$ & -0.0106 & 0.0023 & $3.80 \mathrm{E}--06$ & -0.0051 & 0.0037 & 0.1678 \\
\hline rs17017431 & TRAF5 & 1 & $A$ & $\mathrm{~T}$ & 0.0105 & 0.0023 & $3.80 E-06$ & 0.0022 & 0.0038 & 0.5628 \\
\hline rs10853533 & SLC14A2 & 18 & A & $C$ & 0.0103 & 0.0023 & $6.09 E-06$ & -0.0068 & 0.0043 & 0.1133 \\
\hline rs2115151 & SPATA5 & 4 & A & $\mathrm{T}$ & 0.0103 & 0.0022 & $3.75 \mathrm{E}-06$ & 0.0024 & 0.0041 & 0.5561 \\
\hline rs7015048 & - & 8 & $\mathrm{~T}$ & $C$ & 0.0100 & 0.0015 & $3.16 \mathrm{E}-11$ & -0.0015 & 0.0028 & 0.5864 \\
\hline rs9460424 & - & 6 & $\mathrm{~T}$ & G & -0.0097 & 0.0022 & $9.16 \mathrm{E}-06$ & -0.0053 & 0.0035 & 0.1328 \\
\hline rs4646693 & LRRK1 & 15 & $\mathrm{~T}$ & $C$ & -0.0090 & 0.0020 & $6.80 E-06$ & -0.0001 & 0.0054 & 0.9909 \\
\hline rs8092658 & SLC14A2 & 18 & A & $C$ & -0.0090 & 0.0020 & $6.80 \mathrm{E}-06$ & 0.0001 & 0.0028 & 0.9828 \\
\hline rs1578743 & - & 10 & A & $C$ & 0.0075 & 0.0016 & $1.70 E-06$ & 0.0026 & 0.0029 & 0.3664 \\
\hline rs7973508 & - & 12 & A & G & -0.0073 & 0.0016 & $5.40 \mathrm{E}-06$ & 0.0007 & 0.0030 & 0.8245 \\
\hline rs12464424 & - & 2 & $\mathrm{~T}$ & $C$ & -0.0071 & 0.0016 & $7.47 \mathrm{E}-06$ & 0.0055 & 0.0030 & 0.0651 \\
\hline rs12611788 & GALNT14 & 2 & T & C & -0.0070 & 0.0015 & $5.51 \mathrm{E}-06$ & -0.0062 & 0.0029 & 0.0301 \\
\hline
\end{tabular}

Table 3 Results of metabolic pathway analysis

\begin{tabular}{llll}
\hline Metabolic pathway & Involved Metabolites & P value & Database \\
\hline Alpha linolenic acid and linoleic acid metabolism & Docosapentaenoate (n3 DPA; 22:5n3); & 0.0062 & SMPDB \\
Alpha-linolenic acid metabolism & Linolenate (18:3n3 or 6) & 0.0702 & KEGG \\
Glutathione metabolism & Linolenate (18:3n3 or 6) & 0.0912 & KEGG \\
Beta oxidation of very long chain fatty acids & 5-Oxoproline & 0.0989 & 0.1140 \\
Fructose and mannose metabolism & Acetylcarnitine & 0.1622 & SMPDB \\
Oxidation of branched chain fatty acids & Mannitol & 0.1816 & KEGG \\
Tryptophan metabolism & Acetylcarnitine & SMPB \\
\hline
\end{tabular}

to human intelligence. The only identified metabolic pathway in our study was Alpha linolenic acid and linoleic acid metabolism. Alpha linolenic acid and linoleic acid are long-chain polyunsaturated fatty acids, which are essential nutrients in the development and functioning of the brain [42]. Many related compounds, such as alpha linolenic acid and docosahexaenoic acid, are involved in the rapid growth and development of the infant brain $[43,44]$. Our study thus reinforced the importance of alpha linolenic acid and linoleic acid 
metabolism for human intelligence, providing valuable information for understanding the biological mechanisms related to human intelligence.

The current study has several strengths. First, we implemented a novel MR study design to assess the causal relationships between genetically determined metabolites and human intelligence. By using genetic variants as IVs, the MR approach prevents confounding, reverse causation, and various biases common in observational epidemiological studies. Second, our study provides, indirectly, a comprehensive assessment of the causal effects of metabolites assessed by nontargeted metabolomics on human intelligence. Third, by integrating genomics and metabolomics, our study provides novel insight into the biological mechanisms underlying differences in intelligence.

There are also several limitations that should be noted. First, the GWAS data for intelligence was determined adjusting for socioeconomic status, which was a heritable and correlated secondary trait to intelligence $[29,45]$. The adjustment for socioeconomic status might cause bias in genetic associations with intelligence for some SNPs [46]. Second, our study could not avoid the bias of dynastic effect, which induced a correlation between the environment a child is raised in and their genetic inheritance and almost certainly violated the independence assumption of MR [47, 48]. Within family GWAS data was useful in avoiding the issue of dynastic effects. However, such data was not available at this stage. Third, our study failed to perform the bidirectional MR analysis which was useful in detecting false positive MR results arising from genetic correlation between traits. The reason was that many of the IVs for intelligence were missing in datasets of metabolites. Finally, the MR estimates from non-experimental date could not provide information towards molecular mechanism, further work should be done to determine the roles of metabolites or genetic variants in development of intelligence.

In summary, our study identified multiple metabolites that might have causal effects on human intelligence, among which 5-oxoproline presented significant association signals after Bonferroni correction. The association was shown to be robust by sensitivity analyses. Our study also highlighted that genetic factors (e.g. the EXOSC4 gene) contributed substantially to the variation of metabolite levels and differences in human intelligence. Moreover, our findings suggest that alpha linolenic acid and linoleic acid metabolism might be involved in the biological processes underlying intelligence. Though further evidence from experimental data is needed, our study provides novel clues that would improve our understanding of the biological mechanisms related to human intelligence.

\section{Supplementary Information}

The online version contains supplementary material available at https://doi. org/10.1186/s13041-021-00743-4.

Additional file 1: Fig. S1. Funnel plots for detecting potential pleiotropy. Additional file 2: Fig. S2. Mendelian randomization associations of dihomo-linoleate(20:2n6) on other intelligence-related outcomes from other data sources.

Additional file 3: Fig. S3. Mendelian randomizationassociations of p-acetamidophenylglucuronide on other intelligence-relatedoutcomes from other data sources.

Additional file 4: Table S1. Information of the instrumental variables used for Mendelian randomization estimates. Table S2. Unknown metabolites identified by the Mendelian randomization estimates. Table S3. Genetic predictors for dihomo-linoleate $(20: 2 n 6)$ and their association with Intelligence. Table S4. Genetic predictors for p-acetamidophenylglucuronide and their association with Intelligence.

\section{Abbreviations}

GDM: Genetically determined metabotype; MR: Mendelian randomization; IVW: Inverse-variance weighted; IV: Instrumental variable; GWAS: Genomewide association study; SNP: Single nucleotide polymorphism; InSIDE: Instrument strength independent of direct effect; SMPDB: Small Molecule Pathway Database; KEGG: Kyoto Encyclopedia of Genes and Genomes.

\section{Acknowledgements}

The authors would like to thank the High-Performance Computing Cluster of the First Affiliated Hospital of Xi'an Jiaotong University for data computing support.

\section{Authors' contributions}

$\mathrm{XM}$ and JY were responsible for the study conception and study design. BZ, $L Q, F G$ and YF performed the data collation and statistical analysis. JY and BZ drafted the manuscript. QM and LY were involved in the technical supports. BY and WW contributed to interpretation and editing of the manuscript. All authors read and approved the final manuscript.

Funding

The study was funded by the Clinical Research Award of the First Affiliated Hospital of Xi'an Jiaotong University (Grant No. XJTU1AF-CRF-2017-025).

\section{Availability of data and materials}

Full summary statistics for the 486 metabolites are publicly available at the Metabolomics GWAS Server (http://metabolomics.helmholtz-muenchen.de/ gwas/). GWAS summary statistics for intelligence are download from http:// ctg.cncr.nl/software/summary_statistics.

\section{Ethics approval and consent to participate}

Not applicable.

Consent for publication

All authors read and approved the final manuscript.

\section{Competing interests}

The authors have no conflict of interest.

\section{Author details}

${ }^{1}$ Clinical Research Center, The First Affiliated Hospital of Xi'an Jiaotong

University, Xi'an, China. ${ }^{2}$ Department of Psychiatry, the First Affiliated Hospital 
of Xi'an Jiaotong University, No. 277 Yanta West Road, Xi'an 700061, China.

${ }^{3}$ Center for Brain Science, The First Affiliated Hospital of Xi'an Jiaotong University, Xi'an, China.

Received: 14 May 2020 Accepted: 1 February 2021 Published online: 09 February 2021

\section{References}

1. Deary IJ. Intelligence. Annu Rev Psychol. 2012;63:453-82.

2. Blackwell LS, Trzesniewski KH, Dweck CS. Implicit theories of intelligence predict achievement across an adolescent transition: a longitudinal study and an intervention. Child Dev. 2007;78:246-63.

3. Deary IJ, Strand S, Smith P, Fernandes C. Intelligence and educational achievement. Intelligence. 2007;35:13-21.

4. Burks SV, Carpenter JP, Goette L, Rustichini A. Cognitive skills affect economic preferences, strategic behavior, and job attachment. Proc Natl Acad Sci USA. 2009;106:7745-50.

5. Gottfredson LS. Why g matters: the complexity of everyday life. Intelligence. 1997;24:79-132.

6. Deary I. Why do intelligent people live longer? Nature. 2008;456:175-6.

7. Plomin R, von Stumm S. The new genetics of intelligence. Nat Rev Genet. 2018:19:148-59.

8. Panizzon MS, Vuoksimaa E, Spoon KM, Jacobson KC, Lyons MJ, Franz CE, et al. Genetic and environmental influences of general cognitive ability: is $\mathrm{g}$ a valid latent construct? Intelligence. 2014;43:65-76.

9. Sniekers S, Stringer S, Watanabe K, Jansen PR, Coleman JRI, KrapohI E, et al. Genome-wide association meta-analysis of 78,308 individuals identifies new loci and genes influencing human intelligence. Nat Genet. 2017:49:1107-12.

10. Savage JE, Jansen PR, Stringer S, Watanabe K, Bryois J, de Leeuw CA, et al. Genome-wide association meta-analysis in 269,867 individuals identifies new genetic and functional links to intelligence. Nat Genet. 2018:50:912-9.

11. Nisbett RE, Aronson J, Blair C, Dickens W, Flynn J, Halpern DF, et al. Intelligence: new findings and theoretical developments. Am Psychol. 2012;67:130-59.

12. Dehghan A. Chapter 19 - Linking metabolic phenotyping and genomic information. In: Lindon JC, Nicholson JK, Holmes E, editors. The handbook of metabolic phenotyping. Amsterdam: Elsevier; 2019. p. 561-9.

13. Gieger C, Geistlinger L, Altmaier E, Hrabe de Angelis M, Kronenberg F, Meitinger T, et al. Genetics meets metabolomics: a genome-wide association study of metabolite profiles in human serum. PLoS Genet. 2008;4:1000282

14. Suhre K, Shin S-Y, Petersen A-K, Mohney RP, Meredith D, Wägele B, et al. Human metabolic individuality in biomedical and pharmaceutical research. Nature. 2011;477:54-60.

15. Kettunen J, Tukiainen T, Sarin AP, Ortega-Alonso A, Tikkanen E, Lyytikainen $L P$, et al. Genome-wide association study identifies multiple loci influencing human serum metabolite levels. Nat Genet. 2012;44:269-76.

16. Shin SY, Fauman EB, Petersen AK, Krumsiek J, Santos R, Huang J, et al. An atlas of genetic influences on human blood metabolites. Nat Genet. 2014;46:543-50.

17. Burgess S, Daniel RM, Butterworth AS, Thompson SG, et al. Network Mendelian randomization: using genetic variants as instrumental variables to investigate mediation in causal pathways. Int J Epidemiol. 2015;44:484-95.

18. Burgess S, Small DS, Thompson SG. A review of instrumental variable estimators for Mendelian randomization. Stat Methods Med Res. 2017;26:2333-55.

19. Cheung C-L, Tan KCB, Au PCM, Li GHY, Cheung BMY. Evaluation of GDF15 as a therapeutic target of cardiometabolic diseases in human: a Mendelian randomization study. EBioMedicine. 2019;41:85-90.

20. Sanna S, van Zuydam NR, Mahajan A, Kurilshikov A, Vich Vila A, Vosa U, et al. Causal relationships among the gut microbiome, short-chain fatty acids and metabolic diseases. Nat Genet. 2019;51:600-5.

21. Haycock PC, Burgess S, Nounu A, Zheng J, Okoli GN, et al. Association between telomere length and risk of cancer and non-neoplastic diseases: a Mendelian randomization study. JAMA Oncol. 2017:3:636-51.
22. Evans DM, Davey SG. Mendelian randomization: new applications in the coming age of hypothesis-free causality. Annu Rev Genomics Hum Genet. 2015;16:327-50

23. Burgess S, Butterworth A, Thompson SG. Mendelian randomization analysis with multiple genetic variants using summarized data. Genet Epidemiol. 2013;37:658-65.

24. Bowden J, Davey Smith G, Burgess S. Mendelian randomization with invalid instruments: effect estimation and bias detection through Egger regression. Int J Epidemiol. 2015:44:512-25.

25. Bowden J, Davey Smith G, Haycock PC, Burgess S. Consistent estimation in Mendelian randomization with some invalid instruments using a weighted median estimator. Genet Epidemiol. 2016;40:304-14.

26. Verbanck $M$, Chen $C Y$, Neale B, Do R. Detection of widespread horizontal pleiotropy in causal relationships inferred from Mendelian randomization between complex traits and diseases. Nat Genet. 2018;50:693-8.

27. Hill WD, Marioni RE, Maghzian O, Ritchie SJ, Hagenaars SP, McIntosh AM, et al. A combined analysis of genetically correlated traits identifies 187 loci and a role for neurogenesis and myelination in intelligence. Mol Psychiatry. 2019;24:169-81.

28. Lee JJ, Wedow R, Okbay A, Kong E, Maghzian O, Zacher M, et al. Gene discovery and polygenic prediction from a genome-wide association study of educational attainment in 11 million individuals. Nat Genet. 2018;50:1112-21.

29. Hill WD, Davies NM, Ritchie SJ, Skene NG, Bryois J, Bell S, et al. Genomewide analysis identifies molecular systems and 149 genetic loci associated with income. Nat Commun. 2019;10:1-7.

30. Davies G, Lam M, Harris SE, Trampush JW, Luciano M, Hill WD, et al. Study of 300,486 individuals identifies 148 independent genetic loci influencing general cognitive function. Nat Commun. 2018;9:2098.

31. Chong J, Wishart DS, Xia J. Using metaboanalyst 4.0 for comprehensive and integrative metabolomics data analysis. Curr Protoc Bioinform. 2019;68:86

32. Jewison T, Su Y, Disfany FM, Liang Y, Knox C, Maciejewski A, et al. SMPDB 2.0: big improvements to the small molecule pathway database. Nucleic Acids Res. 2014;42:478-84.

33. Kanehisa M, Goto S, Sato Y, Furumichi M, Tanabe M. KEGG for integration and interpretation of large-scale molecular data sets. Nucleic Acids Res. 2012:40:D109-14.

34. Ristoff E, Larsson A. Inborn errors in the metabolism of glutathione. Orphanet J Rare Dis. 2007:2:16.

35. Rumping L, Vringer E, Houwen RHJ, van Hasselt PM, Jans JJM, VerhoevenDuif NM. Inborn errors of enzymes in glutamate metabolism. J Inherit Metab Dis. 2019;43:200-15.

36. McDougall GJ Jr, Austin-Wells V, Zimmerman T. Utility of nutraceutical products marketed for cognitive and memory enhancement. J Holist Nurs. 2005:23:415-33.

37. Grioli S, Lomeo C, Quattropani MC, Spignoli G, Villardita C. Pyroglutamic acid improves the age associated memory impairment. Fundam Clin Pharmacol. 1990:4:169-73.

38. Hlozek T, Krizek T, Tuma P, Bursova M, Coufal P, Cabala R. Quantification of paracetamol and 5-oxoproline in serum by capillary electrophoresis: implication for clinical toxicology. J Pharm Biomed Anal. 2017;145:616-20.

39. Raijmakers R, Egberts WV, van Venrooij WJ, Pruijn GJM. Protein-protein interactions between human exosome components support the assembly of RNase PH-type subunits into a six-membered PNPase-like Ring. J Mol Biol. 2002;323:653-63.

40. Sharma V, Ounallah-Saad H, Chakraborty D, Hleihil M, Sood R, Barrera I, et al. Local inhibition of PERK enhances memory and reverses agerelated deterioration of cognitive and neuronal properties. J Neurosci. 2018:38:648-58.

41. Ohno M. PERK as a hub of multiple pathogenic pathways leading to memory deficits and neurodegeneration in Alzheimer's disease. Brain Res Bull. 2018;141:72-8.

42. Janssen CI, Kiliaan AJ. Long-chain polyunsaturated fatty acids (LCPUFA) from genesis to senescence: the influence of LCPUFA on neural development, aging, and neurodegeneration. Prog Lipid Res. 2014;53:1-17.

43. Innis SM. Dietary omega 3 fatty acids and the developing brain. Brain Res. 2008;1237:35-43 
44. Simopoulos AP. Evolutionary aspects of diet: the omega-6/omega-3 ratio and the brain. Mol Neurobiol. 2011;44:203-15.

45. Hill WD, Hagenaars SP, Marioni RE, Harris SE, Liewald DCM, Davies G, et al. Molecular genetic contributions to social deprivation and household income in UK Biobank. Curr Biol. 2016;26:3083-9.

46. Aschard H, Vilhjálmsson Bjarni J, Joshi Amit D, Price Alkes L, Kraft P. Adjusting for heritable covariates can bias effect estimates in genome-wide association studies. Am J Hum Genet. 2015;96:329-39.

47. Kong A, Thorleifsson G, Frigge ML, Vilhjalmsson BJ, Young Al, Thorgeirsson $T E$, et al. The nature of nurture: effects of parental genotypes. Science. 2018;359:424-8
48. Koellinger PD, de Vlaming R. Mendelian randomization: the challenge of unobserved environmental confounds. Int J Epidemiol. 2019:48:665-71.

\section{Publisher's Note}

Springer Nature remains neutral with regard to jurisdictional claims in published maps and institutional affiliations.
Ready to submit your research? Choose BMC and benefit from:

- fast, convenient online submission

- thorough peer review by experienced researchers in your field

- rapid publication on acceptance

- support for research data, including large and complex data types

- gold Open Access which fosters wider collaboration and increased citations

- maximum visibility for your research: over $100 \mathrm{M}$ website views per year

At BMC, research is always in progress.

Learn more biomedcentral.com/submissions 